\title{
Mini-symposium
}

\section{Pericardial disease: introduction}

$\mathrm{T}$ his mini-symposium is devoted to the so-called "cinderella of heart disease" - pericardial disease. The management of thise disease, contrary to most cardiac diseases, has scarcely been influenced by recent advances in technology. In many cases the aetiology of pericardial disease is apparently due to the presence of a concomitant disease; however, in patients without an apparent cause the aetiology remains elusive, being idiopathic (in most cases probably being viral in origin) in more than $90 \%$ of cases. Our point of view is that "old" clinical management (history, physical examination, and routine laboratory tests), with a judicious indication of invasive procedures, is the appropriate approach for most patients.
Gaietà Permanyer-Miralda, from Barcelona, comments on the general approach to acute pericardial disease. His point of view is based on a large experience of systematic management of acute pericardial disease for the last 25 years, at the same institution. He also discusses the impact of recent devices (for example, the pericardioscope) or the impact of new diseases (for example, AIDS). Ralph Shabetai, from La Jolla, California, covers the wide spectrum of pericardial effusion, with special emphasis on atypical forms of cardiac tamponade that can only be clinically identified with a high clinical suspicion index. For centuries, cardiac constriction was supposed to be a rather permanent, uniform pattern of pericardial involvement. Jaume Sagristà-Sauleda, from Barcelona, challenges this concept and discusses new patterns of cardiac constriction, from transient to occult constriction.

These presentations aim to provide information which is directly related to the clinical management of patients with pericardial disease. I hope that some of the thoughts from the outstanding contributors of this mini-symposium will help physicians improve their treatment of the "cinderella of heart disease" in the future.

J Soler-Soler Servicio de Cardiologia, Hospital General Universitari, Vall d'Hebron, Barcelona, Spain; soler@vhebron.net

\section{IMAGES IN CARDIOLOGY}

\section{Deep vein thrombosis demonstrated by contrast enhanced helical computed tomographic venography}

\footnotetext{
A 63 year old man was admitted with progressive right lower limb swelling four days after undergoing diagnostic coronary angiography. The procedure was uneventful and showed only minor coronary artery disease. Physical examination showed diffuse bruising over the right groin and swelling of the right lower limb up the thigh. Sonography showed diffuse haematoma over the right groin with no evidence of deep vein thrombosis (DVT). Contrast enhanced computed tomographic (CT) venography with three dimensional reconstruction was performed which revealed the presence of a cresentic thrombus in the proximal part of the right femoral vein with extension into the right superficial femoral vein (arrow).

DVT and fatal pulmonary embolism is a rare but known complication following cardiac catheterisation. It is likely that this patient developed DVT as a consequence of extrinsic compression of the right femoral vein by the haematoma. The presence of haematoma over the soft tissue might lead to a difficulty in identifying the presence of DVT by sonography.

DVT is a common and potentially fatal disease. However, an accurate diagnosis of DVT remains challenging in a significant proportion of patients because of the limitations of conventional non-invasive imaging techniques. The development of helical CT pulmonary angiography and delayed venous imaging (CT venography) has provided a single diagnostic tool that can be used for complete evaluation of thromboembolic disease. As this case shows, CT venography is useful for diagnosing DVT in the event of a negative sonographic result when clinical suspicion is still high.
}

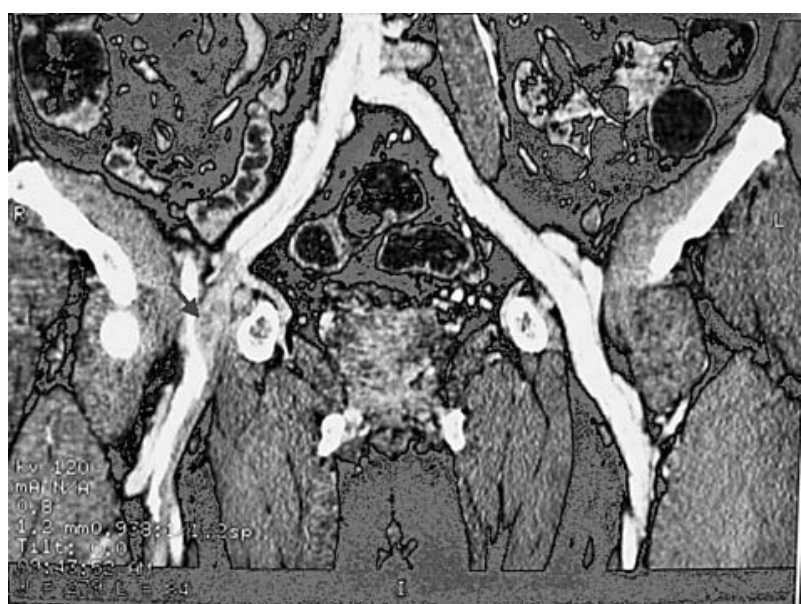

Three dimensional reconstruction of contrast enhanced delayed CT venography of the pelvis and thigh. The right femoral vein is completely filled with clot (arrow) with extension into the superficial femoral vein. The vein appears enlarged in comparison with the right side and there is enhancement of the wall, suggesting perivenous oedema. 